Check for updates

Cite this: RSC Adv., 2017, 7, 45335

\title{
Super-hydrophobic powders obtained by froth flotation: properties and applications
}

\begin{abstract}
Nicolas R. Chevalier (D)
Super-hydrophobic materials offer a wide range of industrially relevant applications such as water-repellent construction materials, surface or textile coatings and oil sorbents. Hydrophobic modification of mineral particles is at the heart of the froth-flotation process. I show here that froth-floated fluorite crystals display super-hydrophobic properties: they form highly stable monolayers on flat and curved ("liquid marble") water surfaces, surround themselves with a gaseous plastron when submerged and selectively disperse and increase the density of oil in an oil-water mixture. I show that resuming the main steps of the flotation process using oleic acid functionalization provides an easy way to render low-solubility calcium salts $\left(\mathrm{CaCO}_{3}, \mathrm{CaF}_{2}\right)$ hydrophobic. I study the potential of hydrophobic crystal monolayers to mitigate freshwater evaporation losses for water management: surprisingly, I find that even very compact, polydisperse crystal layers do not reduce water evaporation rates. I finally present a new method based on hydrophobic modification and X-ray scanning at an air-liquid interface to determine the cleavage planes of ground mineral powders with potential applications in mineralogy and catalysis.
\end{abstract}

Received 28th June 2017

Accepted 18th September 2017

DOI: $10.1039 / \mathrm{c} 7 \mathrm{ra0} 7164 \mathrm{~g}$

rsc.li/rsc-advances

hydrophobic particles, ranging from silane vapor deposition on

\section{Introduction}

The production of durable, abrasion resistant, low-cost superhydrophobic material and coatings is today one of the key challenges of materials science. ${ }^{1}$ The most obvious application of super-hydrophobic materials is water repellency, which is of interest for clothing, building materials, paints, self-cleaning optics, to cite just a few applications. Other highly promising perspectives offered by these materials include viscous drag reduction ${ }^{2}$ on watercraft, anti-icing, ${ }^{3}$ oil-spill remediation, ${ }^{4}$ water decontamination, anti-fouling ${ }^{5}$ and anti-corrosion effects. ${ }^{1}$ Hydrophobic particulate materials have been the focus of considerable attention because powders have an increased surface-to-volume ratio compared to bulk material, thus reinforcing the interfacial effects characteristic of hydrophobic materials. The granular nature of powders adds further interesting physical properties to this material, such as the ability to flow and mold itself into the shape of its container, and porosity with respect to gases. Applications of hydrophobic powders include oil spill remediation, ${ }^{4,6-8}$ water filtration ${ }^{9,10}$ and decontamination, water-repellent filling material in areas that need to be protected from water infiltration or water freezing (e.g., pipes, junction boxes). They are also sold as a toy (kinetic sand or magic sand) for educational puposes. ${ }^{11}$ Because of their many promises and exotic physical properties ${ }^{12,13}$ a variety of methods have been described over the past decades to produce

Laboratoire Matière et Systèmes Complexes, Université Paris Diderot/CNRS UMR 7057, Sorbonne Paris Cité, 10 rue Alice Domon et Léonie Duquet, 75013 Paris, France.E-mail: nicolas.chevalier@univ-paris-diderot.fr silica, ${ }^{14,15}$ polystyrene $^{16}$ or lycopodium ${ }^{13}$ particles to carbonfunctionalization of zeolite ash. ${ }^{17}$

Hydrophobic modification of mineral particles is at the heart of the froth flotation process (Fig. 1), an industrial technique developed to separate minerals from their gangue. ${ }^{18}$ In the first step of froth flotation, the raw ore is ground and mixed with surfactants that selectively adsorb to the mineral particles that one wishes to separate. After surfactant adsorption, gas is injected in the ore-surfactant slurry; the hydrophobic particles adhere to rising air bubbles and concentrate at the liquid surface, forming a froth, which is collected by skimming. The skimmed mineral powder obtained after froth flotation is, de facto, an intrinsically hydrophobic powder (lest the surfactant be removed after collection).

In the froth flotation process, hydrophobicity is a means (to separate the mineral) rather than an end in itself. This has led to the paradoxical situation that many recent surface chemistry papers ${ }^{4,15,20-22}$ describe how to produce hydrophobic lowsolubility mineral particles by addition of surfactant without referring to the fact that these methods have been developed more than a century ago and are currently in use in the mining industry; in fact, froth-floated minerals constitute an abundant and economically interesting source of hydrophobic powders, because the flotation step is already integrated in the industrial process of these minerals. This situation is due in part to the lack of reports documenting the macroscopic hydrophobic behavior (marble formation, monolayer formation, bulk behavior of the powder in water, oil etc.) of mineral ores collected after froth flotation. In surveying the literature, I was 
1- Raw Mineral

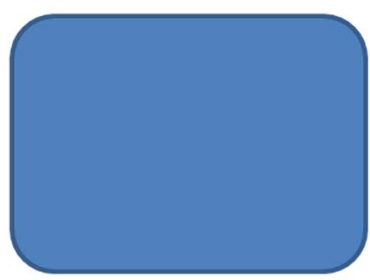

3 - Surfactant Binding

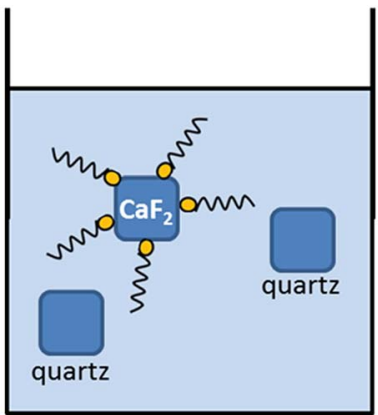

2- Crushing \& Grinding

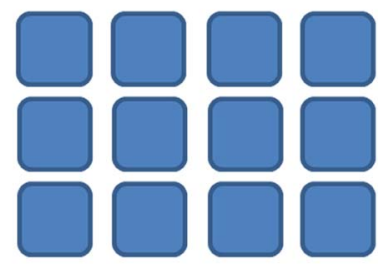

4 - Collecting at surface

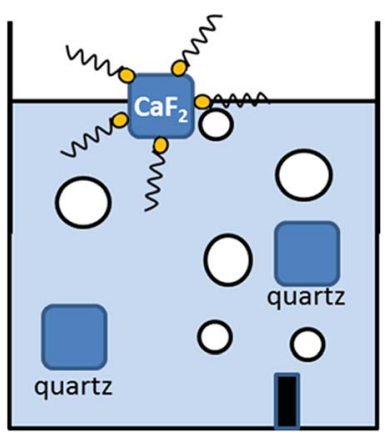

Fig. 1 Principle of froth flotation. Rocks (1) are crushed and ground (2) and dispersed in an aqueous surfactant bath (3). Oleic acid is commonly used to float fluorite. ${ }^{19}$ The negatively charged carboxylic acid groups of the oleate bind to the divalent calcium cations present at the fluorite surface, covering them with a hydrophobic layer. Air bubbles are next injected at the bottom of the bath (4): they attach to the coated fluorite particles and rise, concentrating the mineral at the bath surface. The surface is finally skimmed, washed and dried. The isolated fluorite fraction can be sold as such or further processed.

also surprised to find that although magic (hydrophobic) sand has been used for decades for leisure and educational purposes, ${ }^{23}$ no scientific studies describe or explain the fascinating properties of this material. The main goal of this report is therefore to fill these gaps. I proceed by systematically comparing the wetting properties in water and oil of a nonfloated, pure mineral (fluorite) to those of the same mineral collected after froth-flotation processing. I show that the main steps of froth flotation can easily be reproduced in the laboratory to produce hydrophobic powders. I then present two novel applications of hydrophobic powders: (1) their use as a flexible, self-assembled hydrophobic cover on water to limit evaporation losses, (2) their use in mineralogy and catalysis to determine the dominant cleavage plane of ground minerals. I finally critically discuss my results concerning the hydrophobic properties of froth-floated $\mathrm{CaF}_{2}$ in the light of previous studies that indicated that fluorite could be an intrinsically hydrophobic mineral.

\section{Materials \& methods}

\section{ATR-IR}

Crystals were pressed against the ATR diamond (diameter: 6 $\mathrm{mm}$, refraction index $n=2.4$ ) of a Vertex 70 (Bruker Optics) spectrometer equipped with a monoreflexion ATR MIRacle platform (Pike, US) and an MCT detector. The incidence angle was $45^{\circ}$; the penetration depth of the beam in $\mathrm{CaF}_{2}(n=1.43)$ ranges from 1 to $10 \mu \mathrm{m}$ for the wavelengths shown in Fig. 2a.

\section{SEM}

A small amount of powder was dispersed on metal tape on an aluminium SEM holder. The holder was then gently tapped to remove excess powder. The samples were next electroplated with a conductive carbon layer. Scanning electron microscopy images were acquired on a JEOL JSM-6510 instrument, at 10-15 kV acceleration voltage, secondary or backscattered electron images were collected.

\section{X-Ray powder diffraction}

For standard powder analysis, a small pellet of powder was pressed in a cylindrical hole ( $\sim 1 \mathrm{~cm}$ diameter, $3 \mathrm{~mm}$ deep). For the determination of cleavage planes, the minerals were first dispersed at the air-liquid (glycerol) interface as described in [28]. Samples were then analyzed on a Siemens D5000 diffractometer in $\theta-2 \theta$ mode with an angular step $\Delta \theta=0.01^{\circ}$.

\section{Contact angle measurement}

A small amount of mineral powder was deposited and flattened on a piece of double-sided scotch tape with a spatula. The layer was made thick enough that the deposited droplets did not contact the underlying scotch tape. A $10 \mu \mathrm{L}$ water drop was thereafter deposited on the powder layers. I was unable to deposit less than $10 \mu \mathrm{L}$ on the floated powders because smaller drops adhered to the pipette cone rather than to the powder film. Contact angles were measured with ImageJ. On nontreated synthetic powders $\left(\mathrm{CaCO}_{3}\right.$ and $\left.\mathrm{CaF}_{2}\right)$, the drop completely wetted the powder layer $\left(\theta=0^{\circ}\right)$.

\section{Surface functionalization of $\mathrm{CaF}_{2}$ and $\mathrm{CaCO}_{3}$ salts}

The ratio of oleic acid to mineral mass was determined by estimating the surface-to-volume ratio of the crystals based on their average size $(\sim 10 \mu \mathrm{m}$, see Fig. 5$) .2 \mathrm{~g}$ of precipitated $\mathrm{CaF}_{2}$ (Sigma, 99\% purity, ref. 01123) or $\mathrm{CaCO}_{3}$ (Sigma, ref. 398101) was dispersed in $50 \mathrm{~mL} \mathrm{NaOH}$ solution, $\mathrm{pH} 11$, to which either $1 \mu \mathrm{L}$ (oleic acid to mineral mass ratio $0.44 \mu \mathrm{g} \mathrm{g}^{-1}$ ) or $10 \mu \mathrm{L}$ (ratio $4.4 \mathrm{\mu g} \mathrm{g}^{-1}$ ) of oleic acid (Sigma) was added. At $\mathrm{pH} 11$, the carboxylic acids of the oleic acid are deprotonated and can bind to calcium at the mineral surface. The mineral, lye and oleic acid solution was next stirred for $30 \mathrm{~min}$; the solution was then filtered and the mineral powder dried on a $50{ }^{\circ} \mathrm{C}$ plate for 6 hours. At higher oleic acid to mineral mass ratios $\left(44 \mu \mathrm{g} \mathrm{g}^{-1}\right)$, a significant amount of soap (calcium oleate) also formed and could not be dissociated from the rest of the calcium carbonate powder.

\section{Evaporation rate measurements}

4 circular Petri dishes, $14 \mathrm{~cm}$ in diameter and initially filled with $80 \mathrm{~g}$ of tap water, were placed at room temperature and subject to a light, uniform breeze (air conditioning). Two samples were covered with a monolayer of $\mathrm{CaF}_{2}$ (geological origin, froth floated) by sprinkling and blowing the powder on the liquid surface until it formed a dense layer. Excess powder was blown away from the interface. Evaporation was compared with that 

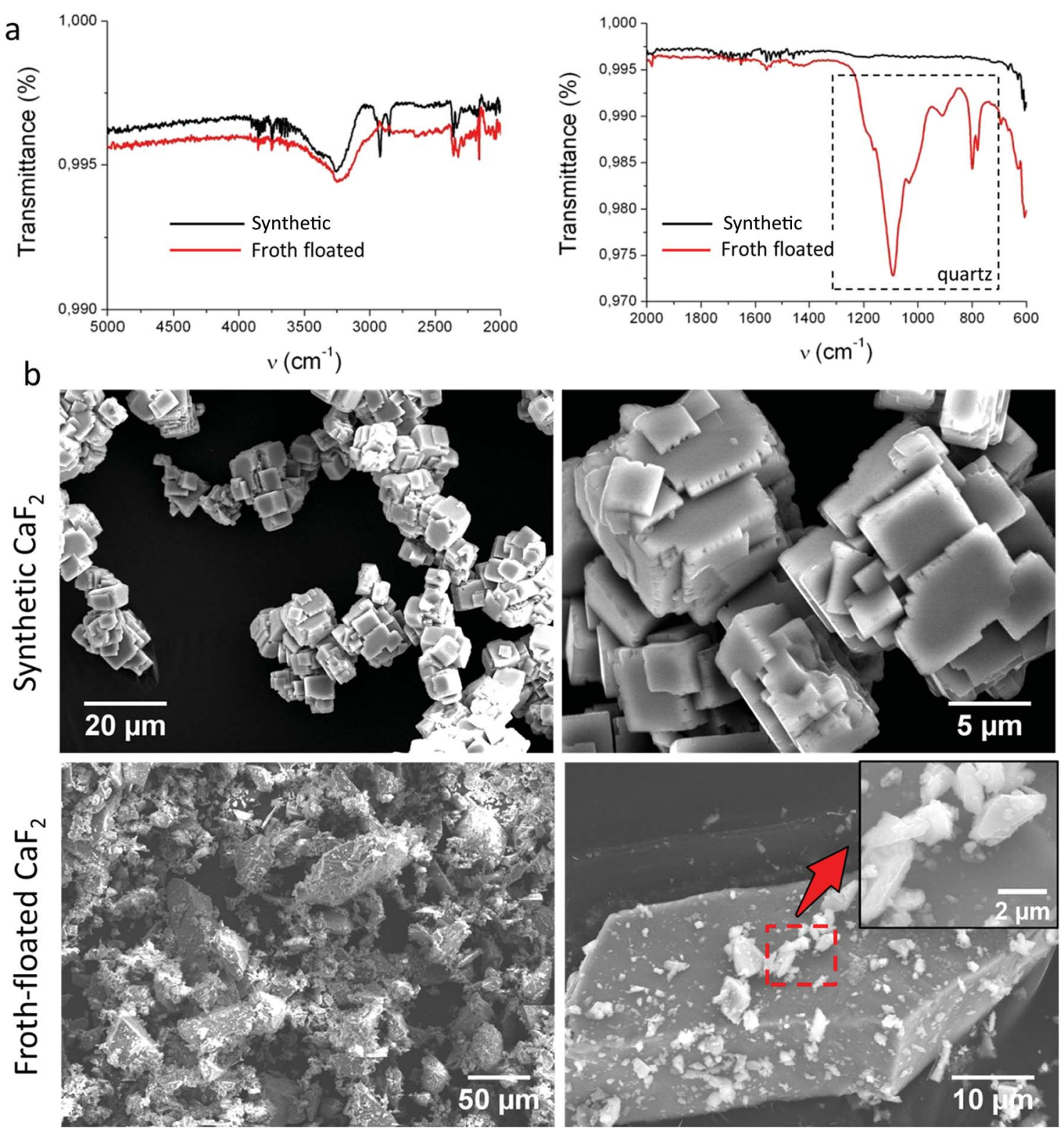

Fig. 2 Chemical and morphological analysis of $\mathrm{CaF}_{2,5}$ and $\mathrm{CaF}_{2, \text { ff }}$ crystals. (a) Compared ATR-IR spectra. Both samples display high IR transparency characteristic of $\mathrm{CaF}_{2}$, although traces of quartz $\left(800-1300 \mathrm{~cm}^{-1}\right.$ ) are found in the geological froth-floated sample. (b) Low and high magnification scanning electron microscopy pictures of $\mathrm{CaF}_{2, \mathrm{~s}}$ (top) and $\mathrm{CaF}_{2, \mathrm{ff}}$ crystals (bottom). The inset shows a magnified view of the granules in the dashed red rectangle.

from a bare water interface (two samples). The mass of water in each dish was measured at different time intervals.

\section{Results}

I examined two fluorite samples: (1) fluorite originating from a geological source, which had an orange hue and had been isolated by froth-flotation, hereafter referred to as $\mathrm{CaF}_{2, \mathrm{ff}},(2)$ pure synthetic fluorite, hereafter referred to as $\mathrm{CaF}_{2, \mathrm{~s}}$, a white powder obtained from the precipitation of $\mathrm{NaF}$ and $\mathrm{CaCl}_{2}$. I first present a brief chemical and morphological analysis to characterize each sample type, and then describe and quantify their wetting behavior.
Chemical and morphological characterization of synthetic and froth-floated fluorite crystals

ATR-IR-spectra and scanning electron microscopy of $\mathrm{CaF}_{2, \mathrm{ff}}$ and $\mathrm{CaF}_{2, \mathrm{~s}}$ are shown in Fig. 2. ATR-IR (Fig. 2a) shows that $\mathrm{CaF}_{2, \text { ff }}$ contained traces of quartz (peaks around $600-1300 \mathrm{~cm}^{-1}$ (ref. 24)). $\mathrm{CaF}_{2, \mathrm{~s}}$ crystals were relatively uniformly sized cubes with well-defined, sharp edges and smooth (100) faces (Fig. 2b, top panels). $\mathrm{CaF}_{2, \mathrm{ff}}$ crystals were strongly polydisperse and their crystalline habit could not be identified morphologically (Fig. 2b, bottom panels). Big $(\sim 50-100 \mu \mathrm{m}) \mathrm{CaF}_{2, \mathrm{ff}}$ appeared covered with small $(\sim 5-10 \mu \mathrm{m})$ adhering $\mathrm{CaF}_{2, \text { ff }}$ particles (Fig. 2b, bottom, inset), yielding an overall "bushy", rough 
morphology. XRD powder diffraction spectra of $\mathrm{CaF}_{2, \mathrm{ff}}$ and $\mathrm{CaF}_{2, \mathrm{~s}}$ sample were identical to that of pure $\mathrm{CaF}_{2}$ as reported in the Mincryst database ${ }^{25}$ (data not shown).

\section{Froth-floated fluorite is ultra-hydrophobic and oleophilic}

Fluorite is almost insoluble in water $\left(K_{\mathrm{d}}=3.910^{-11}\right)$. Like most insoluble ionic salts, $\mathrm{CaF}_{2, \mathrm{~s}}$ dispersed when mixed with water, resulting in a white, turbid solution which became clear as the crystals sedimented. In contrast, when I dipped a spoonful of $\mathrm{CaF}_{2 \text {,ff }}$ (Fig. 3a) in water, the powder dropped to the bottom of the container as one cohesive block (Fig. 3c); it did not disperse. I could easily deform this block to any desired shape.

When I removed the block of powder from water, it was dry (Fig. 3d). As the $\mathrm{CaF}_{2 \text {,ff }}$ powder crossed the interface, a significant fraction remained at the water surface, forming a "skin" (Fig. 3b). The block of powder submerged in water had a metallic shine (Fig. 3c) due to total internal reflection of light, indicating that the powder was surrounded by a film of gas. When the film of gas was destabilized by boiling the solution or by vacuum pumping, the $\mathrm{CaF}_{2, \text { ff }}$ particles dispersed in the solution. This shows that it is the air entrained by the particles as they cross the air-water interface that is responsible for the cohesion of the powder block in water; the fluorite particles in the submerged block are not directly in contact with water. I quantified the wettability of both fluorite types by measuring the contact angle of water drops on films of powder (Fig. 3e, Materials \& methods). I found contact angles of $\sim 156 \pm 4^{\circ}$ ( $n=5$ drops, $\pm \mathrm{SD}$ ) for $\mathrm{CaF}_{2, \mathrm{ff} \cdot}$ On $\mathrm{CaF}_{2, \mathrm{~s}}$, water drops completely wetted the powder substrate. As the contact angle of water on froth-floated $\mathrm{CaF}_{2}$ exceeds $150^{\circ}$, it qualifies as an ultrahydrophobic material. $\mathrm{CaF}_{2 \text {,ff }}$ crystals could wrap themselves around spherical water droplets ("armored drop" or "liquid marble"12,13) and stabilize them even after they were transferred on a hydrophilic (metallic) substrate (Fig. 3f). I further measured capillary rise in small cylindrical pillars of compressed synthetic and floated $\mathrm{CaF}_{2}$ : whereas water quickly rose in the $\mathrm{CaF}_{2, \mathrm{~s}}$ column, I did not observe any capillary rise in the $\mathrm{CaF}_{2, \mathrm{ff}}$ sample (Fig. 3g).

I further examined the behavior of $\mathrm{CaF}_{2, \mathrm{~s}}$ and $\mathrm{CaF}_{2, \mathrm{ff}}$ in binary oil:water solutions (Fig. 4a). After agitation, the synthetic $\mathrm{CaF}_{2, \mathrm{~s}}$ crystals remained in the water phase and gradually sedimented to the bottom of the container. For $\mathrm{CaF}_{2, \mathrm{ff}}$-oil-water ternary mixture, the phase diagram depended on the mineral particle concentration. At low mineral-in-oil concentrations $\left(0.09 \mathrm{~g} \mathrm{~mL}^{-1}\right), \mathrm{CaF}_{2, \mathrm{ff}}$ concentrated and dispersed in the oil phase on top of the water, coloring it orange. When the quantity of $\mathrm{CaF}_{2, \mathrm{ff}}$ was high enough $\left(0.16 \mathrm{~g} \mathrm{~mL}^{-1}\right)$, a fraction of the oil phase sank because the density of the oil- $\mathrm{CaF}_{2, \mathrm{ff}}$ slurry had exceeded that of water. I found that most ( $\sim 95 \%)$ of the oil sank at mineral concentrations of $0.29 \mathrm{~g} \mathrm{~mL}^{-1}$ (Fig. 4a). The calculated average density of the oil- $\mathrm{CaF}_{2, \mathrm{ff}}$ slurry at this
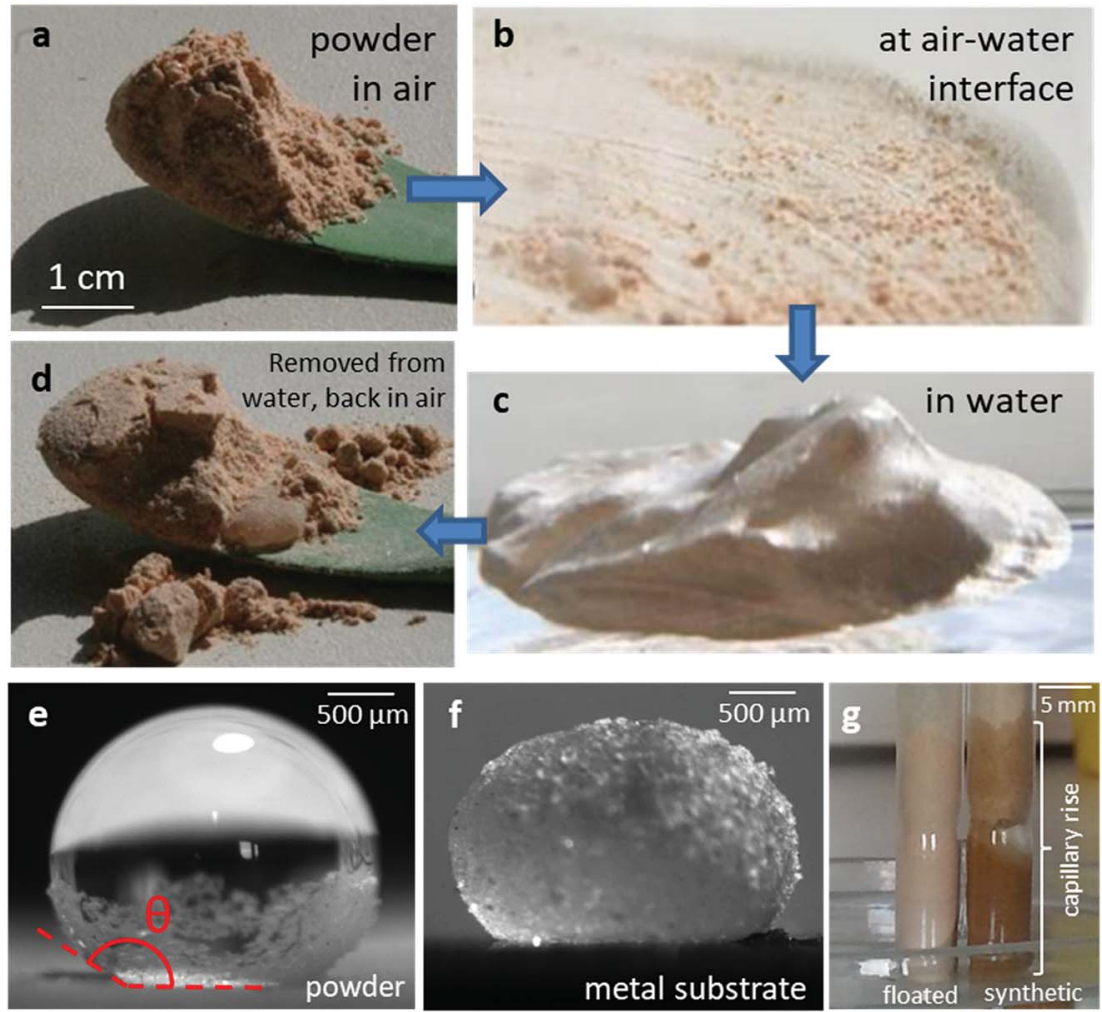

Fig. 3 (a-d) Behavior of froth-floated fluorite powder $\left(\mathrm{CaF}_{2, \mathrm{ff}}\right)$ at the air-water interface, in water, and when removed from water. (e) Contact angle of a water drop $(10 \mu \mathrm{L})$ on a pellet of $\mathrm{CaF}_{2, f f}$. (f) A drop of water $(10 \mu \mathrm{L})$ can be completely covered by CaF $\mathrm{F}_{2, \mathrm{ff}} \mathrm{crystals}$ ("armored drop" or "liquid marble"); this drop remains spherical even when put in contact with a hydrophilic (metal) substrate. (g) Compared capillary rise in pillars of froth-floated and synthetic fluorite. No rise occurs in the froth-floated sample. 
a
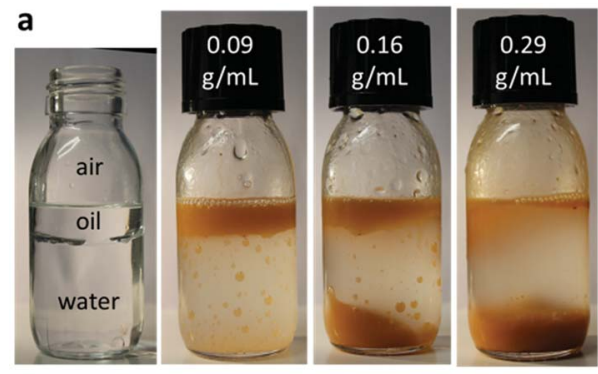

b

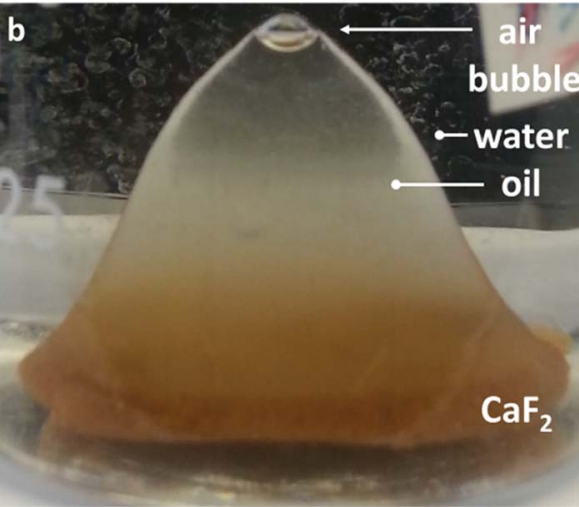

Fig. 4 Behavior of the ternary oil-water- $\mathrm{CaF}_{2, f f}$ system after agitation. (a) Particles are added to $10 \mathrm{~mL}$ of silicone oil (M100, Carl Roth, $\rho=0.96 \mathrm{~g} \mathrm{~cm}^{-3}$ ) floating atop $30 \mathrm{~mL}$ of water at mineral-in-oil concentrations of $0.09 \mathrm{~g} \mathrm{~mL}^{-1}$ (particles in oil, no sinking), $0.16 \mathrm{~g} \mathrm{~mL}^{-1}$ (partial sinking of oil-mineral slurry) and $0.29 \mathrm{~g} \mathrm{~mL}^{-1}$ (almost complete sinking of oil-mineral slurry) of $\mathrm{CaF}_{2}$. (b) After $\mathrm{CaF}_{2}$ had sedimented to the bottom of the sunken oil phase, a portion of the oil could become buoyant again (top, translucent part of the dome) and eventually make its way back to the surface.

concentration is $1.12 \mathrm{~g} \mathrm{~mL}^{-1}$. I also observed that after $\mathrm{CaF}_{2, \mathrm{ff}}$ had sedimented to the bottom of the sunken oil phase, a portion of the oil could become buoyant again and made its way back to the surface (Fig. 4b).

\section{An easy recipe to render low-solubility calcium salts} hydrophobic

The main steps (see Materials \& methods) of the froth flotation process can easily be reproduced in the laboratory, which can be helpful to introduce concepts of hydrophobicity and surface functionalization to a classroom. I used as a starting material synthetic (hydrophilic) $\mathrm{CaF}_{2}$ and $\mathrm{CaCO}_{3}$ crystals and oleic acid as a collector. I used two different ratios of oleic acid to mineral mass: $0.44 \mu \mathrm{g} \mathrm{g}^{-1}$ and $4.4 \mu \mathrm{g} \mathrm{g}^{-1}$ (see Materials \& methods). Compared SEM images of the synthetic $\mathrm{CaF}_{2}$ and $\mathrm{CaCO}_{3}$ powders before and after oleic acid treatment (Fig. 5, top) did not reveal any morphological changes, showing that the surfactant is present at the mineral surface as nanometric layers, not as bulk micrometric aggregates. I found that the synthetic $\mathrm{CaF}_{2}$ and $\mathrm{CaCO}_{3}$ crystals after treatment with oleic acid featured exactly the same macroscopic hydrophobic properties as the floated fluorite sample described above: the surface-functionalized powders could stabilize water drops with a high contact angle (Fig. 5, bottom) and did not disperse in water (Fig. 5, bottom, inset). I now investigate two new applications of hydrophobic powders.

\section{Can water evaporation rates be reduced by covering with a monolayer of hydrophobic particles?}

Across the world, freshwater is valued as a critical natural resource. In countries subject to high seasonal contrast (Australia, India, Africa, etc.), freshwater lakes fill up during the rain season and are used as reservoirs during the dry season. Limiting evaporation loss of freshwater during the dry season is therefore a critical issue. ${ }^{26}$ Several methods have been developed to mitigate freshwater evaporation rates, ranging from Langmuir monolayers, ${ }^{27}$ to thicker chemical films or plastic covers that reduce the exposed surface. I showed (Fig. 2b) that hydrophobic froth-floated mineral particles form stable, micrometer thick films at the air-water interface. Being thicker $(\sim 1-10 \mu \mathrm{m})$ than monolayers $(\sim 10 \mathrm{~nm})$, they are mechanically and chemically more stable with respect to wind, surface waves or UV/bacterial degradation. These particles spread naturally on the surface of water, making them readily applicable to large water reservoirs. A high surface coverage $(>90 \%)$ can be achieved because the $\mathrm{CaF}_{2, \mathrm{ff}}$ particles are polydisperse, so the gaps between large particles can be filled with smaller particles, making the monolayer very compact (see Fig. 6, inset). I measured that only $\sim 6-7 \mathrm{~g}$ of $\mathrm{CaF}_{2 \text {,ff }}$ crystals are required to cover a $1 \mathrm{~m}^{2}$ liquid surface. Surprisingly however, I found that evaporation rates (see Materials \& methods) were not affected by the presence of a compact crystal monolayer (Fig. 6). Average evaporation rates were 30.7 $\pm 0.1 \mathrm{mg} \mathrm{s}^{-1} \mathrm{~m}^{-2}$ for the bare surface and $30.8 \pm 3.4 \mathrm{mg} \mathrm{s}^{-1}$ $\mathrm{m}^{-2}$ for the $\mathrm{CaF}_{2}$ covered surface.

\section{The cleavage plane of minerals can be determined by $\vartheta-2 \vartheta$} XRD scans of froth-floated particles at the air-water interface

When a mineral is ground it breaks along its cleavage planes: the cleavage planes make up most of the physical surface of ground crystals. We have recently found ${ }^{28}$ that crystals deposited at the air-water interface are spontaneously oriented by surface tension forces so that their most extended physical surface lies parallel to the air-water interface. Stabilization of the crystals at the air-water interface is facilitated by hydrophobic modification of the surface of the crystals (e.g., with fatty acid collectors as described above). This suggests a straightforward two-step procedure (Fig. 7) to determine the cleavage plane of any ground mineral powder: (1) surface modification to render the powder hydrophobic so that the crystals can be easily deposited and are stable at a liquid interface (Fig. 7a and b) (2) $\theta-2 \theta$ XRD scan of the crystals at the liquid interface (Fig. 7c and $\mathrm{d}$ ).

I have validated this method for ground froth-floated $\mathrm{CaF}_{2, \mathrm{ff}}$ (Fig. 7d) and for ground $\mathrm{CaCO}_{3} \cdot{ }^{28}$ After the hydrophobic particles are deposited at the air-water interface, the only remaining diffraction peak is (111) for $\mathrm{CaF}_{2}$ and (104) for $\mathrm{CaCO}_{3}$, i.e., the respective main cleavage planes of these minerals. I emphasize that for $\mathrm{CaF}_{2}$, the cleavage plane could not have been determined from the mere examination of the morphology of the 

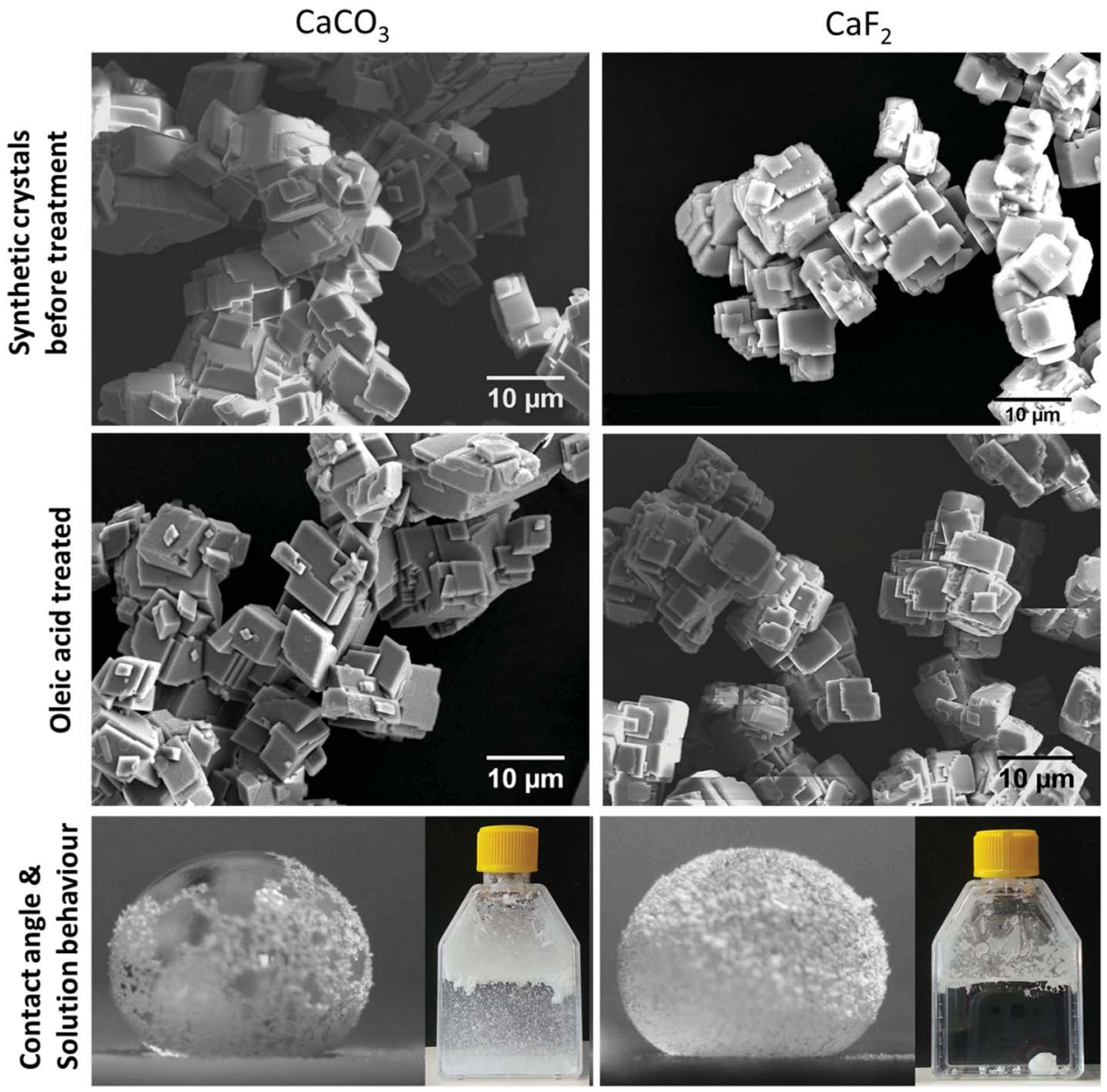

Fig. 5 Oleic acid functionalized synthetic $\mathrm{CaCO}_{3} \& \mathrm{CaF}_{2}$ particles. Top: SEM micrographs of the synthetic $\mathrm{CaCO}_{3} \& \mathrm{CaF}_{2}$ particles before and after treatment with $4.4 \mu \mathrm{g}$ oleic acid per gram of mineral: oleic acid is present as molecular layers at the crystal surface, it does not change the morphology of the samples. Bottom: Hydrophobic behavior of these powders after oleic acid treatment.

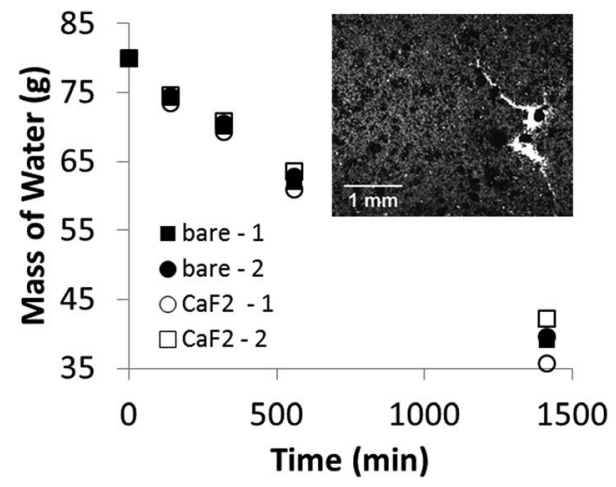

Fig. 6 Effect of $\mathrm{CaF}_{2, f f}$ single crystal layer on evaporation. The residual mass of water is shown as a function of time for four samples. Full symbols: bare water surface (2 samples), open symbols: $\mathrm{CaF}_{2, \mathrm{ff}}$ covered water surface (2 samples). Inset: photograph of the $\mathrm{CaF}_{2, \mathrm{ff}}$ monolayer, a bright spot presenting the water surface beneath due to a fracture in the monolayer is shown to stress the compactness of the mineral layer in the remaining portions of the dish.

crystals (see Fig. 2b, bottom panel): this method therefore provides important, non-trivial information on the physical faces expressed in a ground powder.

\section{Discussion}

I showed that whereas synthetic $\mathrm{CaF}_{2}$ displays the usual hydrophilic properties of an ionic salt, froth-floated $\mathrm{CaF}_{2}$ behaves as a super-hydrophobic material. I documented several enthralling phenomena (Fig. 3 and 4) resulting from the combined granular and hydrophobic nature of this material, such as water drop stabilization, surface film formation, nondispersibility and oil-densification in binary oil-water mixtures. I designed a simple oleic acid functionalization protocol inspired from froth flotation techniques to make synthetic fluorite and calcite crystals hydrophobic (Fig. 5). While the fact that froth-floated minerals are hydrophobic is not new, I could not find any reports documenting the macroscopic properties resulting from this simple surface treatment; furthermore, many authors report on $\mathrm{CaCO}_{3}$ functionalization by fatty acids ${ }^{\mathbf{4}, 15,20-22}$ without apparently being aware that the technics they describe are used in the froth-flotation process. One purpose of this work is therefore to refresh this fact to the community of scientist working on hydrophobic materials. Froth flotation, a technic which has been refined for over a century in the mining industry to separate mineral particles as a function of their affinity to different surfactants, provides 


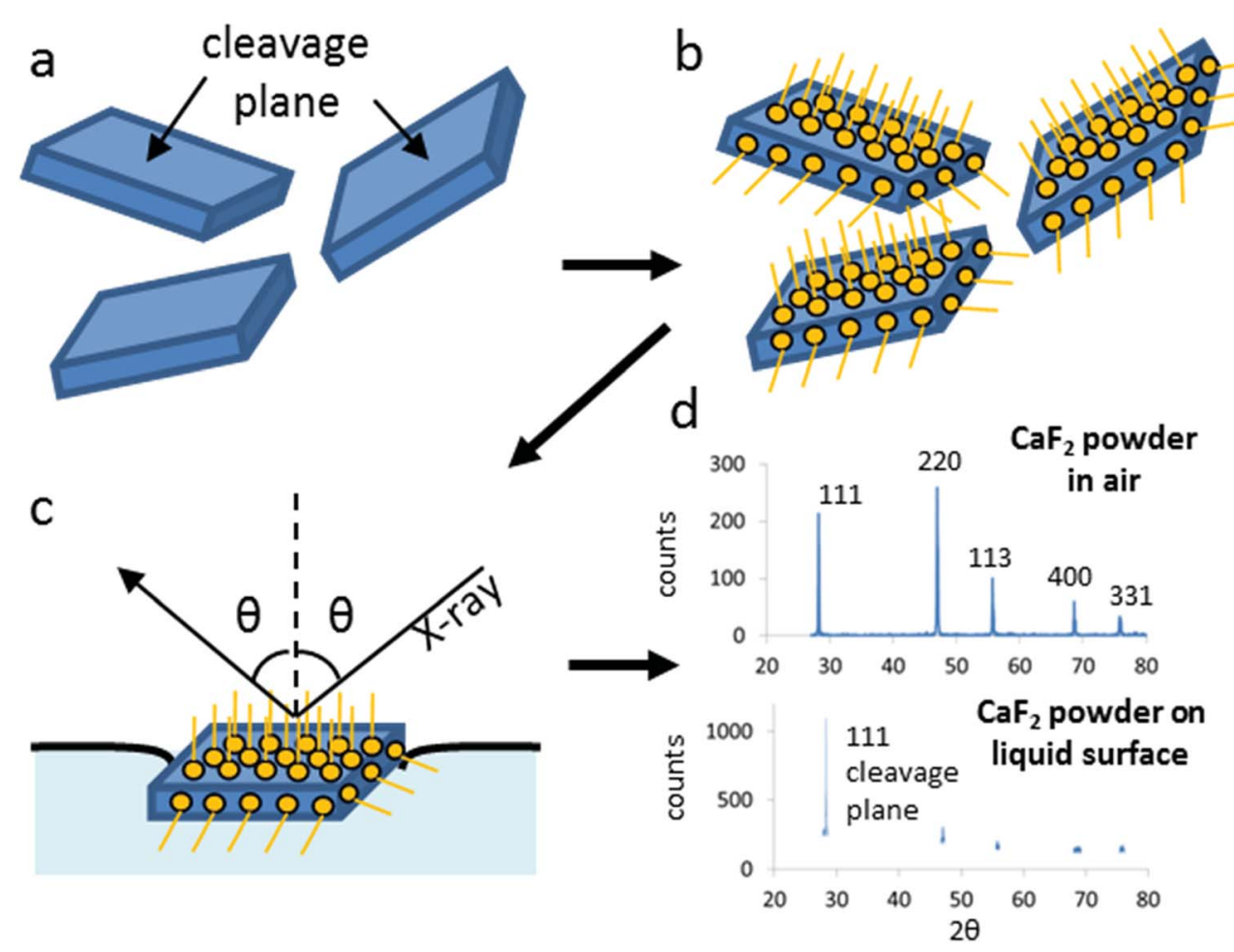

Fig. 7 Two-step procedure to determine the cleavage planes of ground crystal powders. The crystals (a) are covered with surfactant (b) rendering them hydrophobic so they can be deposited and are stable (not sinking) at an air-liquid interface. At the interface, surface tension reorients the crystals so that their cleavage plane lies parallel to the interface (c). A $\theta-2 \theta$ scan of the crystals at the liquid (here glycerol) surface reveals that the only remaining diffraction peak corresponds to the 111 cleavage plane (d).

a straightforward industrial source of hydrophobic mineral powder. I finally examined two novel applications of hydrophobic powders to reduce water evaporation rates (Fig. 6) and determine the cleavage plane of mineral powders (Fig. 7).

Some investigators ${ }^{29,30}$ claimed that fluorite is an inherently hydrophobic mineral based on the fact that water contact angles of cleaved natural fluorite monocrystals (presumably the (111) face) reached up to $100^{\circ}$; in addition, molecular dynamics simulations showed that water is depleted in the vicinity of the (111) $\mathrm{CaF}_{2}$ plane, ${ }^{31}$ which could explain this hydrophobicity. Upon compiling other values of contact angle measurements on native $\mathrm{CaF}_{2},{ }^{30-33} \mathrm{I}$ found that most reported values fall in the range 20 $50^{\circ}$; they do not significantly differ from the contact angle of the freshly cleaved 104 face of $\mathrm{CaCO}_{3}\left(\theta \sim 50^{\circ}\right.$ (ref. 32)), a hydrophilic mineral. Based on my observations on the effects of minute amounts of surfactant, it is possible that some of the very high contact angles of water on cleaved geological fluorite measured by Zawala et $a l^{30}$ may be due to contamination or adsorption of organic material. My observations do not support the view that fluorite is inherently a hydrophobic mineral, because the pure synthetic $\mathrm{CaF}_{2}$ crystals displayed hydrophilic properties.

Laborie et al. ${ }^{16}$ recently reported results on the use of monodisperse micrometric hydrophobically functionalized polystyrene particles to limit water evaporation rates: they found that a single particle layer did not modify the evaporation rate, similarly to what I found here using polydisperse hydrophobic fluorite crystals (Fig. 6). This behavior is due ${ }^{34,35}$ to the fact that the water vapor concentration profile above holes (spaces between crystals) is $3 \mathrm{D}$, contrary to the case of the bare surface, where the vapor profile is stratified (1D). Consequently, the evaporation rate per unit surface trough the micron-sized holes in the monolayer is higher than the average evaporation rate per unit surface of the bare interface; the resulting average evaporation rate through the particle monolayer ends up being equal to that of the bare interface. Achieving evaporation reduction on flat interfaces with hydrophobic particles would therefore require a complementary component like a binder to fill the gaps between the particles; the particles could also be added to existing polymer-based evaporation reduction liquids. ${ }^{36}$

I presented a new method (Fig. 7) to measure the cleavage planes of a mineral. This method provides information on the physical faces expressed in a ground powder and could have important applications in mineralogy and catalysis. It will be interesting to test the methods on minerals where more than one cleavage plane is present. The liquid on which the particles are deposited could additionally be modified (e.g. ethanol, glycerol, oil) to optimize attachment of the particles at the interface and make it applicable to crystals that are soluble in water.

The unique properties of hydrophobic powders (granular, hydrophobic, high surface-to-volume, porous) will no doubt reveal more, as of yet unforeseen applications of this material.

\section{Conflicts of interest}

There are no conflicts to declare. 


\section{Acknowledgements}

I thank Guillaume Wang (MPQ, Paris Diderot) for help with SEM sample preparation, SEM image acquisition and analysis, Hélène Bouquerel (IPGP) for assistance in performing the sieve analysis, Pascale Chenevier (CEA) for providing fluorite samples, Patrick Guenoun (CEA) and Corinne Chevallard (CEA) for access to the X-ray diffractometer and ATR-IR spectrometer, Vincent Fleury (MSC) and Ameya Murukutla (CRI, Paris) for discussions on applications of hydrophobic powders.

\section{Reference}

1 J. T. Simpson, S. R. Hunter and T. Aytug, Superhydrophobic Materials and Coatings: A Review, Rep. Prog. Phys., 2015, 78(8), 86501.

2 G. McHale, M. I. Newton and N. J. Shirtcliffe, Immersed Superhydrophobic Surfaces: Gas Exchange, Slip and Drag Reduction Properties, Soft Matter, 2010, 6(4), 714-719.

3 M. A. Sarshar, C. Swarctz, S. Hunter, J. Simpson and C. H. Choi, Effects of Contact Angle Hysteresis on Ice Adhesion and Growth on Superhydrophobic Surfaces under Dynamic Flow Conditions, Colloid Polym. Sci., 2013, 291(2), 427-435.

4 M. Patowary, K. Pathak and R. Ananthakrishnan, A Facile Preparation of Superhydrophobic and Oleophilic Precipitated Calcium Carbonate Sorbent Powder for Oil Spill Clean-Ups from Water and Land Surfaces, RSC Adv., 2015, 5(97), 79852-79859.

5 A. K. Epstein, T.-S. Wong, R. A. Belisle, E. M. Boggs and J. Aizenberg, Liquid-Infused Structured Surfaces with Exceptional Anti-Biofouling Performance, Proc. Natl. Acad. Sci. U. S. A., 2012, 109(33), 13182-13187.

6 D. Boglaienko and B. Tansel, Science of the Total Environment Gravity Induced Densi Fi Cation of Fl Oating Crude Oil by Granular Materials: Effect of Particle Size and Surface Morphology, Sci. Total Environ., 2016, 556, 146-153.

7 D. Boglaienko and B. Tansel, Instantaneous Stabilization of Floating Oils by Surface Application of Natural Granular Materials (Beach Sand and Limestone), Mar. Pollut. Bull., 2015, 91(1), 107-112.

8 M. Abkarian, S. Protière, J. M. Aristoff and H. A. Stone, Gravity-Induced Encapsulation of Liquids by Destabilization of Granular Rafts, Nat. Commun., 2013, 4, 1895.

9 M. Rashed, Adsorption Technique for the Removal of Organic Pollutants from Water and Wastewater, Org. Pollut.: Monit., Risk Treat., 2013, 167.

10 T. Darmanin and F. Guittard, Recent Advances in the Potential Applications of Bioinspired Superhydrophobic Materials, J. Mater. Chem. A, 2014, 2, 16319.

11 A. Widener, What's That Stuff? Magic Sand And Kinetic Sand, Chem. Eng. News, 2015, 93(12), 41.

12 G. McHale and M. I. Newton, Liquid Marbles: Topical Context within Soft Matter and Recent Progress, Soft Matter, 2015, 11(13), 2530-2546.
13 P. Aussillous and D. Quéré, Liquid Marbles, Nature, 2001, 411(6840), 924-927.

14 B. P. Binks and R. Murakami, Phase Inversion of ParticleStabilized Materials from Foams to Dry Water, Nat. Mater., 2006, 5(11), 865-869.

15 X. Men, B. Ge, P. Li, X. Zhu, X. Shi and Z. Zhang, Facile Fabrication of Superhydrophobic Sand: Potential Advantages for Practical Application in Oil-Water Separation, J. Taiwan Inst. Chem. Eng., 2016, 60, 651-655.

16 B. Laborie, F. Lachaussée, E. Lorenceau and F. Rouyer, How Coatings with Hydrophobic Particles May Change the Drying of Water Droplets: Incompressible Surface versus Porous Media Effects, Soft Matter, 2013, 9(19), 4822-4830.

17 T. Sakthivel, D. L. Reid, I. Goldstein, L. Hench and S. Seal, Hydrophobic High Surface Area Zeolites Derived from Fly Ash for Oil Spill Remediation, Environ. Sci. Technol., 2013, 47(11), 5843-5850.

18 M. Fuerstenau and K. Han, Principles of Mineral Processing; Society for Mining, Metallurgy, and Exploration, 2003.

19 K. Fa, T. Jiang, J. Nalaskowski and J. D. Miller, Interaction Forces between a Calcium Dioleate Sphere and Calcite/ fluorite Surfaces and Their Significance in Flotation, Langmuir, 2003, 19(25), 10523-10530.

20 A. Barhoum, S. M. El-Sheikl, F. Morsy, S. El-Sherbiny, F. Reniers, T. Dufour, M.-P. Delplancke, G. Van Assche and H. Rahier, Preparation and Characterization of UltraHydrophobic Calcium Carbonate Nanoparticles, IOP Conf. Ser.: Mater. Sci. Eng., 2014, 64, 12037.

21 Y. Chen, X. Ji and X. Wang, Facile Synthesis and Characterization of Hydrophobic Vaterite CaCO 3 with Novel Spike-like Morphology via a Solution Route, Mater. Lett., 2010, 64(20), 2184-2187.

$22 \mathrm{Z}$. Hu and Y. Deng, Superhydrophobic Surface Fabricated from Fatty Acid-Modified Precipitated Calcium Carbonate, Ind. Eng. Chem. Res., 2010, 49(12), 5625-5630.

23 E. Vitz, Magic Sand: Modelling the Hydrophobic Effect and Reversed-Phase Liquid Chromatography, J. Chem. Educ., 1990, 67(6), 512.

24 http://lisa.chem.ut.ee/IR_spectra/paint/fillers/quartz/.

$25 \mathrm{http}: / /$ database.iem.ac.ru/mincryst/index.php.

26 J. Frenkel, Evaporation Reduction, UNESCO Arid Zo. Res. XXVII, 1965.

27 G. T. Barnes, The Potential for Monolayers to Reduce the Evaporation of Water from Large Water Storages, Agric. Water Manag., 2008, 95(4), 339-353.

28 N. R. Chevalier and P. Guenoun, Surface Tension Drives the Orientation of Crystals at the Air-Water Interface, J. Phys. Chem. Lett., 2016, 7(14), 2809-2813.

29 B. Jańczuk, J. M. Bruque, M. L. González-Martín and J. M. del Pozo, Wettability and Surface Tension of Fluorite, Colloids Surf., A, 1993, 75(C), 163-168.

30 J. Zawala, Natural Hydrophobicity and Flotation of Fluorite, Physicochem. Probl. Miner. Process., 2007, 41, 5-11.

31 K. Fa, A. V. Nguyen and J. D. Milled, Hydrophobic Attraction as Revealed by AFM Force Measurements and Molecular Dynamics Simulation, J. Phys. Chem. B, 2005, 109(27), 13112-13118. 
32 P. B. Kowalczuk, C. Akkaya, M. Ergun, M. J. Janicki, O. Sahbaz and J. Drzymala, Water Contact Angle on Corresponding Surfaces of Freshly Fractured Fluorite, Calcite and Mica, Physicochem. Probl. Miner. Process., 2017, 53(1), 192-201.

33 Z. Y. Gao, W. Sun, Y. H. Hu and X. W. Liu, Anisotropic Surface Broken Bond Properties and Wettability of Calcite and Fluorite Crystals, Trans. Nonferrous Met. Soc. China, 2012, 22(5), 1203-1208.
34 E. Shahraeeni, P. Lehmann and D. Or, Coupling of Evaporative Fluxes from Drying Porous Surfaces with Air Boundary Layer: Characteristics of Evaporation from Discrete Pores, Water Resour. Res., 2012, 48(9), 1-15.

35 S. Assouline, K. Narkis and D. Or, Evaporation from Partially Covered Water Surfaces, Water Resour. Res., 2010, 46(10), 1-12.

36 M. Wald, Slick Idea Proposed to Stretch Water Supplies, Nature, 2015, 519, 15-16. 